\title{
Effects of meat preheating and wrapping on the levels of polycyclic aromatic hydrocarbons in charcoal-grilled meat.
}

\begin{abstract}
The contamination of polycyclic aromatic hydrocarbons PAHs by thermally treated highprotein foods, such as charcoal-grilled meat products, is due to the generation by direct pyrolysis of food nutrients and the direct deposition of PAHs from smoke produced through incomplete combustion of the thermal agents. This study investigated the effects on the PAH (BaP, BbFln and Fln) contents by using two different types of treatments, preheating (steam and microwave) and wrapping (aluminium and banana leaf) of the meat samples prior to charcoal grilling. PAHs were extracted through tandem solid-phase extraction. The results showed that the applied preheating and wrapping treatments on the samples strongly affect the PAHs levels in the charcoal-grilled meat. No carcinogenic PAHs (BaP and BbFln) were detected in the samples after steam and microwave preheating or aluminium wrapping treatments. Significant reductions of Fln content, of up to $46 \%$ and $81 \%$ in beef and chicken samples, respectively, were observed in the study.
\end{abstract}

Keyword: Polycyclic aromatic hydrocarbons (PAHs); Reduction; Charcoal-grilled meat; Preheating; Wrapping. 